\title{
GUIA EDUCATIVO DE APOIO A FAMILIARES E CUIDADORES DE IDOSOS COM ALZHEIMER: VALIDAÇÃO DE CONTEÚDO
}

\author{
EDUCATIONAL SUPPORT GUIDE FOR FAMILY \\ MEMBERS AND CAREGIVERS OF THE ELDERLY WITH \\ ALZHEIMER'S: CONTENT VALIDATION
}

\section{GUÍA EDUCATIVA PARA APOYAR A FAMILIARES Y CUIDADORES DE ANCIANOS CON ALZHEIMER: VALIDACIÓN DE CONTENIDO}

\author{
Isabella Soares Pinheiro Pinto ${ }^{1}$ \\ Elizabeth Teixeira ${ }^{2}$ \\ Aldalice Aguiar de Souza ${ }^{3}$ \\ Anete Leda de Oliveira ${ }^{4}$ \\ Aline Lima de Souza 5 \\ Karolina Dessimoni Victoria ${ }^{6}$
}

Como citar este artigo: Pinto ISP, Teixeira E, Souza AA, Oliveira AL, Souza AL, Victoria KD. Guia educativo de apoio a familiares e cuidadores de idosos com Alzheimer: validação de conteúdo. Rev baiana enferm. 2021;35:e42533.

Objetivo: criar e validar um guia educativo como recurso tecnológico no apoio a cuidadores e familiares de idosos com Alzheimer. Método: pesquisa metodológica guiada pelo modelo de Pasquali para validação de conteúdo, desenvolvida em quatro etapas: revisão da literatura, na busca da síntese do conhecimento; construção da primeira versão e produção do guia educativo; validação de conteúdo por juízes-especialistas; construção da segunda versão. Os dados obtidos foram analisados estatisticamente com base no índice de concordância mínima de $80 \%$. Revisão do guia para elaboração da versão final na quarta etapa. Estudo realizado em âmbito nacional, por meio digital, atingindo regiões Norte, Nordeste e Centro-Oeste do Brasil. Resultados: participaram da validação 15 juízes-especialistas. O conteúdo do guia educativo obteve índice de validade de conteúdo global de 0,90 em uma única rodada. Conclusão: o guia educativo revelou-se adequado e válido como recurso tecnológico no apoio a familiares e cuidadores de idosos com Alzheimer.

Descritores: Doença de Alzheimer. Assistência a Idosos. Qualidade de Vida. Estudo de Validação. Educação em Saúde. Tecnologia Educacional.

Objective: to create and validate an educational guide as a technological resource to support caregivers and family members of the elderly with Alzheimer's. Method: methodological research guided by Pasquali's model for content validation, developed in four stages: literature review, in the search for knowledge synthesis; construction of the first

\footnotetext{
Enfermeira. Pesquisadora Independente. Manaus, Amazonas, Brasil. ispp.enfl6@uea.edu.br. https://orcid.org/0000-0002-2193-9733.

Enfermeira. Doutora em Ciências. Professora Visitante da Universidade do Estado do Amazonas. Manaus, Amazonas, Brasil. https://orcid.org/0000-0002-540 I-8 I05. Enfermeira. Doutora em Enfermagem. Professora Adjunta da Universidade do Estado do Amazonas. Manaus, Amazonas, Brasil. https://orcid.org/0000-00023002-4578.

Enfermeira. Especialista em Gerenciamento em Serviços de Enfermagem. Professora da Universidade do Estado do Amazonas. Manaus, Amazonas, Brasil. https://orcid.org/0000-0001-7835-5985

Enfermeira. Instituto de Medicina Social, Universidade do Estado do Rio de Janeiro. Manaus, Amazonas, Brasil. https://orcid.org/0000-0002-7545-9380,

Enfermeira. Pesquisadora Independente. Manaus, Amazonas, Brasil. https://orcid.org/0000-0003-0654-6832.
} 
version and production of the educational guide; content validation by expert judges; construction of the second version. The data obtained were statistically analyzed based on the minimum agreement index of 80\%. Review of the guide for drafting the final version in the fourth stage. A study conducted nationwide, through digital means, reaching the North, Northeast and Midwest regions of Brazil. Results: 15 expert judges participated in the validation. The content of the educational guide obtained a global content validity index of 0.90 in a single round. Conclusion: the educational guide proved to be adequate and valid as a technological resource in supporting family members and caregivers of elderly people with Alzheimer's.

Descriptors: Alzheimer Disease. Old Age Assistance. Quality of Life. Validation Study. Health Education. Educational Technology.

Objetivo: crear y validar una guía educativa como recurso tecnológico de apoyo a cuidadores y familiares de ancianos con Alzheimer. Método: investigación metodológica guiada por el modelo de validación de contenido de Pasquali, desarrollado en cuatro etapas: revisión de la literatura, en la búsqueda de sintesis de conocimiento; construcción de la primera versión y producción de la guía educativa; validación de contenido por jueces expertos; construcción de la segunda versión. Los datos obtenidos fueron analizados estadísticamente en base al índice minimo de concordancia del 80\%. Revisión de la guía para la redacción de la versión final en la cuarta etapa. Un estudio realizado a nivel nacional, a través de medios digitales, llegando a las regiones Norte, Nordeste y Medio Oeste de Brasil. Resultados: 15 jueces expertos participaron en la validación. El contenido de la guía educativa obtuvo un indice global de validez de contenido de 0,90 en una sola ronda. Conclusión: la guía educativa demostró ser adecuada y válida como recurso tecnológico en el apoyo a familiares y cuidadores de ancianos con Alzheimer.

Descriptores: Enfermedad de Alzheimer. Asistencia a los Ancianos. Calidad de Vida. Estudio de Validación. Educación en Salud. Tecnología Educacional.

\section{Introdução}

As Doenças Crônicas não Transmissíveis (DCNT) representam importante problema de saúde pública no mundo, atingem cerca de 60\% das mortes e apresentam crescimento gradativo em países considerados de baixa-média e baixa renda ${ }^{(1)}$.

Na população idosa, as DCNT podem acometer os indivíduos ao longo do seu processo de envelhecimento, acarretando diversas dificuldades, como perda da independência e da capacidade de realizar atividades básicas cotidianas ${ }^{(2)}$. Como exemplo, destacam-se as demências, que afetam não somente o indivíduo acometido, mas se estende a todo o leito familiar e a sociedade, causando um grande impacto psicossocial e econômico ${ }^{(3)}$.

Dentre as demências descritas na literatura, a Doença de Alzheimer (DA) é a que mais atinge a população de 60 anos ou mais ${ }^{(4)}$, acometendo cerca de 35,6 milhões de pessoas em âmbito global. Sua progressão para o ano de 2050 é de aproximadamente 65,7 milhões de casos ${ }^{(1)}$. A DA é uma doença cerebral degenerativa primária de etiologia desconhecida, com aspectos neuropatológicos e neuroquímicos, que acomete múltiplas funções corticais e envolve a compreensão, a linguagem, o comportamento e a memória (CID-102) $)^{(3)}$.

A DA é silenciosa e acomete até os mais preparados, fragilizando o conjunto familiar por completo, ao desencadear inúmeros sentimentos no cuidador, como preocupação, tristeza, remorso entre outros. A família torna-se a principal fonte de prestação de cuidados com as pessoas que vivem com a DA ${ }^{(5)}$. Isto ocorre devido à preocupação ou responsabilidade legal familiar, ainda que haja um déficit de conhecimento sobre os cuidados a serem prestados a essas pessoas ${ }^{(5)}$. Nesse sentido, emergiu o interesse de produzir uma tecnologia educacional (TE) que pudesse contribuir com a prestação de cuidados com o idoso portador da Doença de Alzheimer, com base na necessidade de uma produção validada que proporcionasse não somente a melhoria dos cuidados ofertados, mas também a qualidade de vida do indivíduo e de seus cuidadores.

No contexto da prática de saúde e enfermagem, as tecnologias educacionais vêm sendo produzidas, mas a grande maioria não é validada nem avaliada. Os materiais educativos impressos, do tipo fôlderes, cartazes, cartilhas, manuais, 
cadernos de orientação ou apostilas, costumam ser disponibilizados à população antes de serem testados e nem sempre (ou quase nunca) são submetidos a um processo de validação. Isso ocorre principalmente devido ao fato de muitos profissionais de saúde e enfermagem não conhecerem esse processo ${ }^{(6)}$.

Com base no exposto, surgiram as seguintes questões: Um guia sobre DA produzido com base na síntese e tradução do conhecimento disponível na literatura, após validação de conteúdo, revela-se adequado para pessoas que convivem e cuidam de idosos com Alzheimer? Quais as sugestões de juízes especialistas sobre o guia?

Este estudo tem o objetivo de criar e validar um guia educativo como recurso tecnológico no apoio a cuidadores e familiares de idosos com Alzheimer.

\section{Método}

Trata-se de uma pesquisa metodológica ${ }^{(7)}$, com abordagem quantitativa, desenvolvida em quatro etapas (Figura 1).

Figura 1 - Fluxograma das etapas da pesquisa metodológica de validação do guia educativo

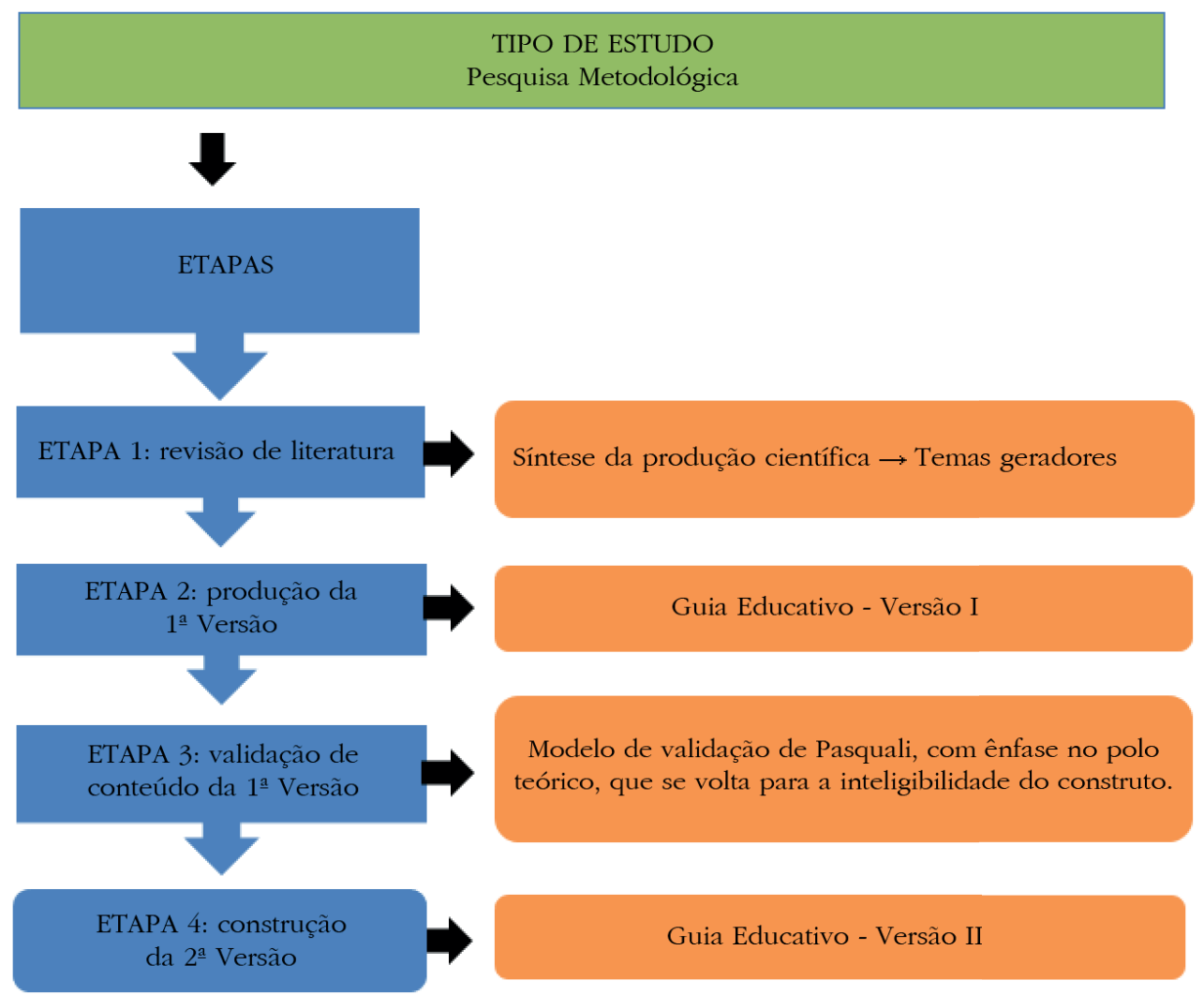

Fonte: Elaboração própria.

A etapa de revisão ocorreu no período de agosto a dezembro de 2019; a primeira versão da tecnologia educacional, foi produzida de janeiro a maio de 2020; a validação do conteúdo da tecnologia educacional, por juízes especialistas ${ }^{(8)}$, aconteceu de junho a agosto de 2020; e a construção da segunda versão da tecnologia educacional, deu-se entre agosto e setembro de 2020.
Para a revisão da literatura, formulou-se a seguinte questão: Quais informações são necessárias para apoiar cuidadores e familiares de idosos com Alzheimer, para garantir qualidade de vida em contexto domiciliar? Na sequência, foram definidos os descritores: Doença de Alzheimer; Assistência a Idosos; Qualidade de Vida; Estudo de Validação; Educação em Saúde; Tecnologia 
Educacional. Para a combinação dos descritores em saúde, foram utilizados os operadores booleanos $A N D$ e Or. Os critérios de inclusão foram: artigos científicos completos, disponíveis de forma gratuita, publicados nos últimos dez anos (2009-2020), nos idiomas português, inglês e espanhol. Foram critérios de exclusão: teses, dissertações, editoriais, publicações duplicadas e aquelas que não respondessem à questão de pesquisa formulada. Após a seleção, foi realizada a leitura dos textos completos e a síntese da produção científica, com vistas à identificação dos temas geradores.

Para a produção da primeira versão do guia educativo, tomaram-se como referência os temas geradores identificados. Após a seleção dos conteúdos e das ilustrações, foram feitas a edição e diagramação, de acordo com critérios relacionados ao conteúdo (cuidados com idosos com Alzheimer) e à estrutura-organização (formato impresso), com atenção especial aos aspectos linguagem, leiaute, design e sensibilidade cultural.

Para a validação de conteúdo, os juízes especialistas foram selecionados por meio de critérios de inclusão - expertise no âmbito da TE (Tecnologia Educacional), por consulta na plataforma Lattes) - e de exclusão - não responder a contato eletrônico por 20 dias no período da coleta de dados. Quanto à definição do número de juízes especialistas, considerou-se uma amostra entre 17 e 24 juízes $^{(9)}$. Assim, foram identificados 24 especialistas. Após o convite, 21 aceitaram participar do estudo. Após o envio do instrumento, 15 devolveram no prazo estabelecido de 20 dias.

A coleta de dados foi realizada via e-mail, com o encaminhamento de um e-mail-convite. Após resposta, envio de novo e-mail com uma via da TE em PDF, uma via do instrumento e uma via do Termo de Consentimento Livre e Esclarecido (TCLE), por meio de um formulário na plataforma Google Forms.

Aplicou-se o questionário validado e organizado conforme a escala de Likert ${ }^{(10)}$, com perguntas referentes à avaliação do conteúdo da TE, com três partes: identificação, orientação de preenchimento e escala tipo Likert. Cada juiz-especialista expressou sua avaliação em graus de 1 a 4, sendo 1 (Totalmente adequado), 2 (Adequado), 3 (Parcialmente adequado) e 4 (Inadequado), de modo a obter o Índice de Validade de Conteúdo (IVC). Este foi calculado por meio do somatório de concordância dos itens marcados como "1" e "2", dividido pelo total de respostas.

Após a coleta, os dados foram inseridos em uma planilha no Microsoft Excel®, versão 2013, e posteriormente tratados estatisticamente. A análise quantitativa pautou-se em um IVC igual ou superior a 80\%. Para a produção da segunda versão do guia educativo, consideraram-se as sugestões encaminhadas pelos juízes.

Conforme as recomendações éticas do Ministério da Saúde ${ }^{(11)}$, na Resolução no 466/12, inciso II, alínea 5, cada juiz assinou o TCLE. Esta pesquisa é um subprojeto de um projeto integrado, aprovado pelo Comitê de Ética em Pesquisa do Centro Universitário Luterano de Manaus, conforme Certificado de Apresentação de Apreciação Ética (CAAE) n ${ }^{\circ}$ 88932418.3.0000.5014.

\section{Resultados}

Na revisão da literatura (Etapa 1), obtiveram-se 16 artigos, que foram codificados por numeração arábica (1, 2, $3 \ldots$ 16). Após leitura exaustiva dos artigos, foram listados 13 temas significativos para compor o guia educativo (Quadro 1).

Quadro 1 - Temas Geradores segundo identificação dos artigos em que foram citados

(continua)

\begin{tabular}{|l|l|}
\hline Temas Geradores & Identificação dos artigos em que foram citados \\
\hline 1 - Definição sobre a Doença de Alzheimer & $1,2,3,5,6,7,8,9,10,12,15,16$ \\
\hline 2 - Classificações e estágios & $1,2,3,5,6,16$ \\
\hline 3 - Sinais e sintomas & $1,2,3,5,6,7,9,10,12,14$ \\
\hline 4 - Autonomia & $1,3,6,10$ \\
\hline 5 - Comunicação & $1,2,9,12$
\end{tabular}


Quadro 1 - Temas Geradores segundo identificação dos artigos em que foram citados

(conclusão)

\begin{tabular}{|l|l|}
\hline Temas Geradores & Identificação dos artigos em que foram citados \\
\hline 6 - Segurança e proteção & $1,3,5$ \\
\hline 7 - Atividade física e lazer & $1,4,13$ \\
\hline 8 - Grupo de apoio & $1,2,3,8$ \\
\hline 9 - Infraestrutura do domić́lio & 1,10 \\
\hline 10 - Higiene & $1,2,3,4,5,7$ \\
\hline 11 - Nutrição e hidratação & 1,4 \\
\hline 12 - Medicações & $1,2,3,4,11$ \\
\hline 13 - Integridade da pele/ Mudança de decúbito & 1,4 \\
\hline
\end{tabular}

Fonte: Elaboração própria.

A primeira versão do guia (Etapa 2) foi baseada nos temas identificados na revisão de literatura. Foram produzidos textos no formato de diálogo entre a personagem principal, intitulada "Dona Lu", que aborda os temas sobre cuidados com o idoso com a Doença de Alzheimer, e a personagem "Dona Maria". Após a criação do conteúdo, tanto textual como imagético, a primeira versão, intitulada "Guia para Familiares e Cuidadores: sobre a Doença de Alzheimer", continha 88 páginas. Foi desenvolvida no programa CANVA. A primeira versão foi disponibilizada aos juízes especialistas no formato digital para avaliação.

Para o processo de validação de conteúdo (Etapa 3), foram selecionados 15 juízes especialistas, com idades de 31 a 57 anos (média 44,66 anos), das áreas de Medicina, Enfermagem e Psicologia (Tabela 1).

Tabela 1 - Perfil sociodemográfico dos juízes-especialistas. Manaus, Amazonas, Brasil - 2020. $(\mathrm{N}=15)$

\begin{tabular}{|c|c|c|}
\hline Variáveis & $\mathbf{n}$ & $\%$ \\
\hline \multicolumn{3}{|l|}{ Gênero } \\
\hline Masculino & 1 & 6,67 \\
\hline Feminino & 14 & 93,33 \\
\hline Idade - Média & 44,66 anos & \\
\hline \multicolumn{3}{|l|}{ Faixa etária } \\
\hline $30-35$ & 3 & 20 \\
\hline $36-40$ & 3 & 20 \\
\hline $41-45$ & 2 & 13,33 \\
\hline $46-50$ & 2 & 13,33 \\
\hline $51 \mathrm{ou}+$ & 5 & 33,33 \\
\hline \multicolumn{3}{|l|}{ Região/local de residência } \\
\hline Norte & 12 & 80 \\
\hline Nordeste & 1 & 6,67 \\
\hline Sudeste & - & - \\
\hline Sul & - & - \\
\hline Centro-Oeste & 2 & 13,33 \\
\hline \multicolumn{3}{|l|}{ Área de formação } \\
\hline Enfermagem & 13 & 86,67 \\
\hline Psicologia & 1 & 6,67 \\
\hline Medicina & 1 & 6,67 \\
\hline \multicolumn{3}{|l|}{ Ocupação } \\
\hline Docente universitário & 8 & 53,33 \\
\hline Enfermeiro assistencial & 4 & 26,67 \\
\hline Médico assistencial & 1 & 6,67 \\
\hline Secretária Municipal de Saúde do Idoso & 2 & 13,33 \\
\hline
\end{tabular}


Tabela 1 - Perfil sociodemográfico dos juízes-especialistas. Manaus, Amazonas, Brasil - 2020. $(\mathrm{N}=15)$

\begin{tabular}{l|cc}
\hline Variáveis & $\mathbf{n}$ & $\mathbf{\%}$ \\
\hline Tempo de exercício profissional & & 26,67 \\
5-10 anos & 4 & 20 \\
$11-15$ anos & 3 & 6,67 \\
$16-20$ anos & 1 & 13,33 \\
$21-25$ anos & 2 & 33,33 \\
$26-30$ anos & 5 & \\
Titulação & & 26,67 \\
Especialista & 4 & 40 \\
Mestre & 6 & 33,33 \\
Doutor & 5 & $\mathbf{1 0 0}$ \\
Total & $\mathbf{1 5}$ & \\
\hline
\end{tabular}

Fonte: Elaboração própria.

Nota: Sinal convencional utilizado:

- Dado numérico igual a zero não resultante de arredondamento.

Os aspectos objetivos, estrutura e apresentação e ainda relevância, que se espera alcançar com a utilização do guia, estão expostos em três blocos.

No "Bloco 1 - Objetivos", que se refere aos objetivos que se propõe a atingir com a utilização do guia e das metas, aplicou-se o questionário validado e organizado conforme a escala de Likert $^{(10)}$, com perguntas referentes à avaliação do conteúdo da TE, com três partes: identificação, orientação de preenchimento e escala tipo Likert. Cada juiz-especialista expressou sua avaliação em graus de 1 a 4, sendo 1 Totalmente Adequado (TA), 2 Adequado (A), 3 Parcialmente Adequado (PA), 4 Inadequado (I).

Obtiveram-se 49 (65,33\%) avaliações para TA, 23 (30,67\%) para (A), 3 (4\%) para (PA) e nenhum Inadequado. De acordo com as opções de respostas dadas pelos juízes, os escores TA e A totalizaram 72, o que correspondeu a um Índice de Validade de Conteúdo (IVC) de 0,96.

Em relação ao "Bloco 2 - Estrutura e apresentação", que se refere à apresentação das orientações do guia, estrutura, aparência, coerência e formatação, obtiveram-se 95 (52,78\%) para TA, 58 (32,22\%) para A, 22 (12,22\%) para PA, e 5 (2,78\%) para I. De acordo com a avaliação dos juízes, PA e A totalizaram, juntos, 153, representando 85\% das respostas válidas. O IVC foi de 0,85.
Em relação ao "Bloco 3 - Relevância", que se refere à significação da tecnologia educacional produzida, obteve-se 50 (66,67\%) marcações para TA, 21 (28,00\%) marcações para A, 4 (5,33\%) marcações para PA e nenhuma marcação para I. De acordo com as opções de resposta dadas pelos juízes, os escores TA e A totalizaram 71, o que equivale a 94,67\% das respostas válidas. Este bloco alcançou IVC de 0,95.

A TE obteve 194 (58,78\%) marcações TA e 102 (30,90\%) marcações A, totalizando 296 marcações. Obtiveram-se 29 (8,78\%) PA e 5 (1,5\%) I. Atingiu-se um IVC geral de 0,90. Em relação ao IVC, dentre os 22 itens, obtiveram-se 21 itens acima de 0,70 e 1 abaixo de 0,70.

Os juízes-especialistas ofereceram as seguintes sugestões: reduzir o número de páginas do guia, por meio da compilação de assuntos; incluir imagens onde há quantidade excessiva de texto; atentar para os cuidados que são específicos de enfermagem; revisar ortografia e formatação; aplicar normas da língua portuguesa; substituir termos técnicos por termos coloquiais ou inserir a definição. Concluíram que todos os profissionais podem utilizá-lo, mas ele está direcionado principalmente para o cuidador familiar; destacaram a grande importância para o público a que se destina e para o grande alcance de conteúdo. 
A segunda versão do guia educativo (Etapa 4) ficou assim organizada: agradecimentos, sumário, apresentação, Parte 1 A Doença de Alzheimer, com as seções 1.1 Esquecimento e 1.2 Fases da doença; Parte 2 O que é importante saber sobre a Doença de Alzheimer, com as seções 2.1 Memória, 2.2 Música, 2.3 Comunicação, 2.4 Cuidados e Higiene Pessoal, 2.5 Vestuário,
2.6 Eliminações, 2.7 Mudança de decúbito, 2.8 Medicação, 2.9 Hidratação, 2.10 Alimentação, 2.11 Estrutura Física da Residência, 2.12 Proteção e Segurança, 2.13 Socialização, 2.14 Rede de Apoio, 2.15 Atividade Física e Lazer, 2.16 Sono e Repouso, Palavras finais e Referências. Esta versão ficou com 51 páginas.

A Figura 2 apresenta a capa da versão final.

Figura 2 - Capa da Tecnologia Educacional Guia para familiares e cuidadores sobre a Doença de Alzheimer

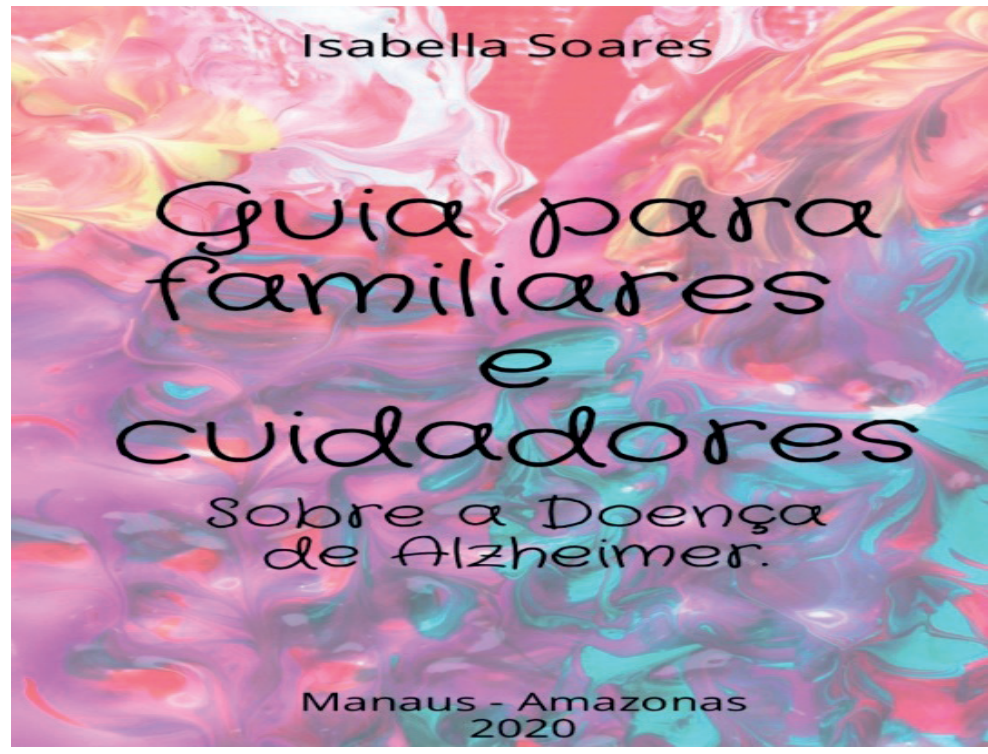

Fonte: Elaboração própria.

\section{Discussão}

A revisão de literatura possibilitou extrair informações sobre os principais temas relacionados à DA e às demandas de cuidado com o idoso, além da construção de um guia de apoio para seus familiares e cuidadores. Os 12 temas selecionados para o guia ilustrado incluíram o conceito da DA, as principais manifestações, suas fases e cuidados primordiais relacionados às alterações cognitivas existentes, de acordo com a evolução da doença.

Estudo aponta que o desenvolvimento de TEs orientadas por revisões de literatura científica proporcionam o uso de conceitos e princípios que fortalecem o alcance dos objetivos do processo educativo esperado. Além disso, o estudo identificou que apenas um terço das pesquisas de TE em idosos utilizou fundamentação científica no processo de construção ${ }^{(12)}$.
Pesquisa $^{(5)}$ reconhece que a família é a principal fonte de prestação de cuidados, devido à preocupação ou responsabilidade legal familiar, ainda que haja um déficit de conhecimento sobre os cuidados a serem prestados às pessoas com DA. Estudos realizados com cuidadores de idosos com necessidades de cuidado revelam que a família é a fonte social de cuidado mais importante para esses indivíduos. Destacam, ainda, que a família desenvolve-se emocional, mental e espiritualmente em espaços inter-relacionais de conflitos, e que, em situações de adoecimento, os integrantes familiares apoiam-se habitualmente, para enfrentar e superar as dificuldades vivenciadas ${ }^{(13-14)}$.

Nesta direção, observa-se a importância das relações de cuidado entre a família e o idoso, a família do idoso e o cuidador e do cuidador com o idoso e o contexto familiar. Deste modo, ressalta-se que a prestação de cuidados, tanto do familiar quanto do cuidador, deve estar respaldada na 
ética, no respeito e no afeto, a fim de estabelecer formas de relacionamento mútuo, atendendo às necessidades impostas pelas reais situações de doença e bem-estar do idoso com DA ${ }^{(15)}$.

O guia construído teve a pretensão de auxiliar principalmente familiares e cuidadores informais, ao trazer uma linguagem coloquial e um diálogo interativo com o leitor, de forma a deixar a leitura mais fácil e compreensiva, e utilizar, em sua grande parte, ilustrações autoexplicativas.

No que concerne à validação da primeira versão desse guia, os juízes especialistas ressaltaram a importância do direcionamento para o cuidador/familiar, conforme descreveram: "[...] de grande importância para o público a que se destina, com um grande alcance. Conteúdo maravilhoso" e "Todos os profissionais podem utilizá-lo, mas ele está muito direcionado para o cuidador familiar". O guia obteve um IVC global dos juízes-especialistas de 0,90, apresentando a validação de seu conteúdo comparando-o com outros estudos nesse âmbito da construção e validação de um produto ${ }^{(16-18)}$.

A construção do guia observou a orientação de estudo ${ }^{(10)}$ sobre escalas de mensuração de atitudes, no qual a produção de TE impressas exige o cumprimento de etapas e percursos, como: levantamento de evidências científicas; definição do objetivo e finalidades da TE; seleção do público alvo da TE; escolha do formato da TE (guia, folder, folheto, manual etc.); definição dos conteúdos e ilustrações; planejamento das ações; e, finalmente, a elaboração do "piloto" da TE.

Após a validação, as sugestões dos juízes especialistas foram listadas e analisadas, resultando nas seguintes alterações na segunda versão do guia: redução do número de páginas, revisão ortográfica, síntese dos conteúdos abordados, troca de termos técnicos por similares ou adição de seu conceito. Esta última alteração resultou do entendimento de que, pelo fato de ser uma tecnologia educacional, seria preciso analisar o público-alvo e o atual contexto em que estava inserido. Foi necessário criar oportunidades, para que cuidadores e familiares conhecessem a doença, para compreender o paciente e, consequentemente, as possibilidades de atuação (seguras e eficazes) nesse paciente ${ }^{(4)}$.
No guia, a opção pelo uso do diálogo como gênero textual foi proposital, pensando no próprio conceito da educação em saúde. Visou-se a relação direta do educador com o educando, no mesmo nível, tendo em vista que o educando participa da sua autonomia, do desenvolvimento do seu próprio conhecimento. Há, assim, não somente a transferência de informação sobre determinado assunto, mas o progresso desse indivíduo $^{(19)}$.

Importante informar que os resultados de estudo $^{(20)}$ mostraram que, na maioria das vezes, o conhecimento e as informações dos cuidadores sobre a doença de Alzheimer eram adquiridos por meio de informações fornecidas pela mídia, o que acabava gerando um tipo de assistência errônea. Além disso, no caso desse estudo, 65\% dos cuidadores relataram não ter recebido nenhum tipo de orientação da equipe de saúde sobre os cuidados necessários e/ou sobre a doença e somente 50\% dos portadores da Doença de Alzheimer recebiam a assistência de algum tipo de profissional da equipe de saúde em ambiente domiciliar. Assim, vê-se a necessidade de disponibilizar a esse público uma tecnologia validada acerca da temática, para embasar não somente o cuidado prestado pelo cuidador, mas também auxiliar profissionais da área da saúde a prestar a educação em saúde para essa população.

O enfermeiro, como parte da equipe multidisciplinar de saúde, pode oferecer aos cuidadores subsídios para a instrumentalização do cuidado e orientar a adaptação desses no contexto domiciliar, referente à progressiva dependência do idoso consequente à doença de Alzheimer, tanto nas atividades mais complexas como em necessidades básicas ${ }^{(21)}$. Assim, a inclusão da TE chega para auxiliar na promoção da educação em saúde, não somente para o indivíduo, mas também para a sociedade.

Aponta-se como dificuldade, neste estudo, a difícil tarefa de seleção dos especialistas e o retorno das respostas on-line no processo de validação da TE. Como fatores limitantes, destaca-se a escassez de estudos recentes no âmbito das TEs para idosos com DA e a não participação do público-alvo do guia na etapa de validação do conteúdo, havendo perspectivas para pesquisas posteriores. 
Embora este estudo tenha alcançado resultados potencialmente positivos para a aplicabilidade do guia, são necessários estudos futuros que possam avaliar cuidadosamente o uso desta TE com o público-alvo.

\section{Conclusão}

O guia educativo criado como recurso tecnológico no apoio a cuidadores e familiares de idosos com Alzheimer foi considerado estatisticamente válido pelos juízes especialistas que participaram da etapa de validação. O guia ilustrado será registrado e disponibilizado em meio impresso. Assim, no contexto da educação em saúde, a TE está adequada e poderá contribuir com o conhecimento e a prática do cuidado junto aos familiares e cuidadores de idosos acometidos com a Doença de Alzheimer, bem como servirá de suporte para os enfermeiros e demais profissionais de saúde na mediação do cuidado.

Ademais, compreende-se que as TEs estruturadas com base no conhecimento científico e validadas, são ferramentas dinamizadoras, criativas e valiosas para a transformação do processo de saúde e doença dos indivíduos. Neste sentido, possibilitam a promoção do cuidado e qualidade de vida aos idosos e a seus cuidadores.

\section{Colaborações:}

1 - concepção, projeto, análise e interpretação dos dados: Isabella Soares Pinheiro Pinto e Elizabeth Teixeira;

2 - redação do artigo e revisão crítica relevante do conteúdo intelectual: Isabella Soares Pinheiro Pinto e Elizabeth Teixeira;

3 - aprovação final da versão a ser publicada: Isabella Soares Pinheiro Pinto, Elizabeth Teixeira, Aldalice Aguiar de Souza, Anete Leda de Oliveira, Aline Lima de Souza e Karolina Dessimoni Victoria.

\section{Referências}

1. Organização das Nações Unidas. Alzheimer: mais de 35 milhões de pessoas sofrem com demência, número que triplicará até 2050 [Internet]. Brasília (DF); 2014 [cited 2019 Aug 20]. Available from: https://brasil.un.org/pt-br/78626oms-numero-de-pessoas-afetadas-por-demenciatriplicara-no-mundo-ate-2050

2. Carvalho JA, Escobar KAA. Cuidador de idosos: um estudo sobre o perfil dos cuidadores de idosos do programa de assistência domiciliar (PAD) da Associação dos Aposentados e Pensionistas de Volta Redonda - AAP-VR. Rev Cient ITPAC [Internet]. 2015 [cited 2019 Aug 21];8(1):1-13. Available from: https://www.itpac.br/arquivos/ Revista/76/Artigo_6.pdf

3. Mendes CFM, Santos ALS. O cuidado na doença de Alzheimer: as representações sociais dos cuidadores familiares. Saúde soc. 2016;25(1): 121-32. DOI: https://doi.org/10.1590/S0104-129 02015142591

4. Falcão D, Braz M, Garcia C, Santos G, Yassuda M, Cachioni M, et al. Atenção psicogerontológica aos cuidadores familiares de idosos com doença de Alzheimer. Psicol, Saúde Doenças. 2018;19(2):377-89. DOI: http://dx.doi.org/10.15309/18psd190217

5. Gonçalves FCA, Lima ICS. Alzheimer e os desafios dos cuidados de enfermagem ao idoso e ao seu cuidador familiar. Rev Pesqui cuid fundam online. 2020;12:1274-82. DOI: https://doi.org/ 10.9789/2175-5361.rpcfo.v12.7971

6. Teixeira E, Medeiros HP, Nietsche EA. Tecnologias Cuidativo-Educacionais. Porto Alegre (RS): Moriá; 2013.

7. Polit DF, Beck CT. Fundamentos de pesquisa em enfermagem: avaliação e evidência para a prática da enfermagem. 9a ed. Porto Alegre (RS): Artmed; 2019.

8. Andrade LZC, Freitas DT, Holanda GF, Silva VM, Lopes MVO, Araújo TL. Desenvolvimento e validação de jogo educativo: medida da pressão arterial. Rev enferm UERJ [Internet]. 2012 [cited 2019 Aug 6];20(3):323-7. Available from: https://www.e-publicacoes.uerj.br/index.php/ enfermagemuerj/article/view/1201/2877

9. Medeiros RK, Ferreira JMA, Pinto DPSR, Vitor AF, Santos VEP, Barichello E. Modelo de validação de conteúdo de Pasquali nas pesquisas em Enfermagem. Rev Enf Ref. 2015;IV(4):127-35. DOI: http://dx.doi.org/10.12707/RIV14009

10. Oliveira TMV. Escalas de mensuração de atitudes: Thurstone, Osgood, Stapel, Likert, 
Guttman. Alpert. FECAP [Internet]. 2001 [cited 2020 Jun 23];2(2):1-21. Available from: https:// pesquisa-eaesp.fgv.br/sites/gvpesquisa.fgv.br/ files/arquivos/veludo_escalas_de_mensuracao_ de_atitudes_thurstone_osgood_stapel_likert_ guttman_alpert.pdf

11. Brasil. Ministério da Saúde. Conselho Nacional de Saúde. Resolução n ${ }^{\circ}$ 466, de 12 de dezembro de 2012. Aprova as diretrizes e normas regulamentadoras de pesquisas envolvendo seres humanos. Brasília (DF); 2012 [cited 2019 Dec 25]. Available from: http://conselho.saude. gov.br/resolucoes/2012/Reso466.pdf

12. Sá GGM, Silva FL, Santos AMR, Nolêto JS, Gouveia MTO, Nogueira LT. Tecnologias desenvolvidas para a educação em saúde de idosos na comunidade: revisão integrativa da literatura. Rev Latino-Am Enfermagem. 2019;27:e3186. DOI: http://dx.doi.org/10.1590/ $1518-8345.3171 .3186$

13. Cardoso RSS, Sá SPC, Domingos AM, Sabóia VM, Maia TN, Padilha JMFO, et al. Educational technology: a facilitating instrument for the elderly care. Rev Bras Enferm. 2018;71(suppl 2):786-92. DOI: https://doi.org/10. 1590/0034-7167-2017-0129

14. Couto AM, Castro EAB, Caldas CP. Vivências de ser cuidador familiar de idosos dependentes no ambiente domiciliar. Rev Rene [Internet]. 2016 [cited 2020 Nov 17];17(1):76-85. Available from: http://200.129.29.202/index.php/rene/article/ view/2624/2011

15. Savieto RM, Leão ER. Assistência em Enfermagem e Jean Watson: uma reflexão sobre a empatia. Esc Anna Nery Rev Enferm [Internet]. 2016 [cited 2020 Nov 17];20(1):198-202. Available from: http://www.scielo.br/pdf/ean/v20n1/14148145-ean-20-01-0198.pdf
16. Alexandre NMC, Coluci MZO. Content validity in the development and adaptation processes of measurement instruments. Ciênc Saúde Coletiva. 2011;16(7):3061-8. DOI: http://dx.doi.org/10.1590/ S1413- 81232011000800006

17. Oliveira SC, Lopes MVO, Fernandes AFC. Development and validation of an educational booklet for healthy eating during pregnancy. Rev Latino-Am Enfermagem. 2014;22(4):611-20. DOI: http://dx.doi.org/10.1590/0104- 1169.3313.2459

18. Silva RA, Ximenes LB, Cruz AG, Serra MA, Araújo MF, Andrade LM, et al. Sexual activity of people with spinal cord injury: development and validation of an educational booklet. Acta Paul Enferm. 2018;31(3):255-64. DOI: http://dx.doi. org/10.1590/1982-0194201800037

19. Oliveira SC, Lopes MVO, Fernandes AFC. Development and validation of an educational booklet for healthy eating during pregnancy. Rev Latino-Am Enfermagem. 2014;22(4):611-20. DOI: http://dx.doi.org/10.1590/0104- 1169.3313.2459

20. Leite SS, Áfio ACE, Carvalho LV, Silva JM, Almeida PC, Pagliuca LMF. Construction and validation of an Educational Content Validation Instrument in Health. Rev Bras Enferm. 2018;71(4):1635-41. DOI: https://doi. org/10.1590/0034-7167-2017-0648

21. Moura PSL, Miranda NF, Rangel LC. As fases da doença de Alzheimer e os cuidados necessários a serem implementados pelo cuidador. Rev Interdiscip Pensamento Científ. 2015;1(2). DOI: 10.20951/2446-6778/v1n2a13

Recebido: 25 de novembro de 2020

Aprovado: 20 de junho de 2021

Publicado: 4 de agosto de 2021

A Revista Baiana de Enfermagem utiliza a Licença Creative Commons - Atribuição-NãoComercial 4.0 Internacional. https://creativecommons.org/licenses/by-nc/4.0/

Este artigo é de acesso aberto distribuído sob os termos da Licença Creative Commons (CC BY-NC). Esta licença permite que outros remixem, adaptem e criem a partir do seu trabalho para fins não comerciais. Embora os novos trabalhos tenham de lhe atribuir o devido crédito e não possam ser usados para fins comerciais, os usuários não têm de licenciar esses trabalhos derivados sob os mesmos termos. 